\title{
Clinical Characteristics of Dizziness Associated with Acute Peripheral Facial Palsy
}

\author{
Kudamo Song ${ }^{1 *}$, Sehun Chang ${ }^{2 *}$, Jun Lee ${ }^{1}$, Sun Ae Shin ${ }^{3}$, and Ho Yun Lee ${ }^{1}$ \\ ${ }^{1}$ Departments of Otorhinolaryngology-Head and Neck Surgery, ${ }^{2}$ Neurosurgery, Eulji University School of Medicine, Daejeon, \\ ${ }^{3}$ Department of Medical Science, Chungnam National University, Daejeon, Korea
}

\author{
Received December 22, 2017 \\ Revised February 2, 2018 \\ Accepted February 2, 2018
}

\author{
Address for correspondence \\ Ho Yun Lee, MD, PhD \\ Department of Otorhinolaryngology- \\ Head and Neck Surgery, \\ Eulji University Medical Center, \\ Eulji University School of Medicine, \\ 95 Dunsanseo-ro, Seo-gu, \\ Daejeon 35233, Korea \\ Tel +82-42-611-3133 \\ Fax +82-42-611-3136 \\ E-mail hoyun1004@gmail.com \\ *These authors contributed equally to \\ this work.
}

Background and Objectives: We aimed to assess the clinical significance of dizziness associated with acute peripheral facial palsy (APFP). Subjects and Methods: Medical records of patients who visited an otorhinolaryngology clinic at a university hospital and were admitted for treatment of APFP between 2014 and 2016 were thoroughly reviewed. Results: In total, $15.3 \%(n=15)$ of patients had dizziness. Continuous, rotatory dizziness without exacerbating factors was most common and frequently accompanied by nausea/vomiting. Dizziness disappeared within 1 week during the hospitalization period. Patients with Ramsay Hunt syndrome (31.0\%) had dizziness more frequently than those with Bell's palsy (8.7\%). In addition, higher hearing thresholds and pain around the ear was reported more often in dizzy patients $(p<0.05)$. Logistic regression analysis revealed that the initial House-Brackmann grade of facial paralysis was solely associated with final recovery, but dizziness was not associated with prognosis. Conclusions: Patients with APFP may have transient dizziness in the early stage, which may be more frequently accompanied by worse hearing thresholds and/or pain around the ear. However, these symptoms including dizziness seem to be unrelated to final prognosis.

J Audiol Otol 2018;22(3):148-153

KEY WORDS: Acute peripheral facial palsy · Bell's palsy · Dizziness · Prognosis · Ramsay Hunt syndrome.

\section{Introduction}

Acute peripheral facial paralysis (APFP) refers to acute flaccid paralysis of ipsilateral facial expression muscles in the absence of central lesions [1]. The most common diagnoses leading to APFP include idiopathic Bell's palsy (BP) and Ramsay Hunt syndrome (RHS). The annual incidence of BP is $11.5-53.3$ persons per 100,000 , and the reactivation of herpes simplex virus type 1 is one of the most common causes. In addition, mite-transmitted infections such as infections with Borrelia burgdorferi in endemic areas or those with varicella-zoster virus (VZV) can also cause BP without skin lesions [2-5]. RHS, which results in facial palsy and skin rash in the ear, is caused by the reactivation of latent VZV in the geniculate ganglion; this occurs most often in patients in their $20 \mathrm{~s}$

This is an Open Access article distributed under the terms of the Creative Commons Attribution Non-Commercial License (http://creativecommons.org/licenses/by-nc/4.0/) which permits unrestricted non-commercial use, distribution, and reproduction in any medium, provided the original work is properly cited. and 30s.

Approximately $70 \%$ of patients with BP experience spontaneous recovery within 6 months even without treatment [5]. However, a recent guideline strongly recommends that steroids be prescribed within $72 \mathrm{~h}$ for patients over the age of 16 years [5]. In addition, patients with severe facial palsy should be treated with combined steroid-antiviral treatment [6]. The prognosis of RHS is worse than that of BP; without appropriate treatment, only $20 \%$ of patients do achieve complete recovery [7]. In general, combined steroid-antiviral treatment is used for treatment of RHS.

Aside from facial paralysis, patients with APFP (both BP and RHS) may experience various other symptoms including pain around the ear, facial numbness, change in taste, eye dryness, hearing disturbance, and dizziness [8]. According to the authors' clinical experience, patients with APFP may become very anxious in response to dizziness, as they often worry about it being a sign of a central brain lesion. Although there are many studies aiming to elucidate the prognostic factors of APFP, to 
the best of our knowledge, there are few studies investigating the clinical features and prognosis of APFP-associated dizziness.

For RHS, it has been suggested that the vestibular nerve and/or labyrinth are involved in the accompanying dizziness [9]. A three-dimensional fluid-attenuated inversion recovery magnetic resonance imaging (MRI) study revealed that the pre-contrast hyperintense signal in the cochlea, vestibule, and the vestibulocochlear nerve of RHS patients was correlated with presence of dizziness [10]. In addition, the different aspects of vestibular impairment and recovery were compared between RHS and other inner ear diseases such as vestibular neuritis (VN) or sudden deafness with vertigo [11,12]. In contrast, the characteristics of dizziness in BP have rarely been studied.

In this study, we assessed the characteristics of APFP-associated dizziness by analyzing data of patients who had been treated for APFP with the aim of applying this information in the clinical setting.

\section{Subjects and Methods}

\section{Ethical considerations}

The Institutional Review Board of Eulji of University Hospital approved this study (IRB number: 2017-09-012). The board granted a waiver of written informed consent because of the retrospective nature of the study.

\section{Patients}

Data from patients who visited the otolaryngology outpatient clinic or emergency room (ER) at Eulji University Hospital for treatment of APFP and who were admitted to hospital for 1 week from 2014 to 2016 were analyzed in the study. Patient age, sex, APFP laterality, recurrence, degree of facial paralysis, diabetes, hypertension, and presence of pain around the ear were documented in addition to presence or absence of dizziness. If vesicles in the ear or oropharynx occurred before or during treatment, the patients were assigned to the RHS group; all other cases were assigned to the BP group.

The enrolled patients consisted of 53 men and 45 women with a mean \pm standard deviation age of $44 \pm 15.9$ years (range: 10-77 years); 49 had left-sided and 49 had right-sided facial palsy. The mean period from onset to treatment was $2.32 \pm 2.24$ days (range: $0-6)$. Among the patients, $5.1 \%(n=$ 5) had diabetes and $14.3 \%(n=14)$ had hypertension. Two patients had previous history of BP. Regarding final diagnosis, 69 patients had BP and 29 had RHS.

The initial degree of facial nerve paralysis was $3.01 \pm 0.753$ according to the House-Brackmann (HB) scale (range: 2-5).
In total, $43.9 \%$ of patients $(n=43)$ had pain around the ear. The mean hearing thresholds in the affected side was $18.6 \pm 19.01$ $\mathrm{dB}$, and this was significantly worse than that of the non-affected side $(11.96 \pm 10.10 \mathrm{~dB})(p<0.001)$.

\section{Otoneurologic evaluation and bedside examination}

Pure-tone audiometry, cervical vestibular-evoked myogenic potential (cVEMP) test, and electroneuronography (ENoG) were conducted in all patients. Pure-tone average was calculated using the mean of hearing thresholds at $500 \mathrm{~Hz}, 1,2$, and $3 \mathrm{kHz}$. For cVEMP tests, the latencies and amplitudes of the p13 and n23 waves were recorded with Navigator Pro software (Bio-logic Systems Corp, Mundelein, IL, USA) [13]. cVEMP asymmetry ratio was calculated using the following formula: (amplitudes difference/sum of amplitude) $\times 100$. ENoG was performed one time within three days from admission to hospital. Compound muscle action potential from the frontalis, orbicularis oculi, nasalis, orbicularis oris, and mentalis were recorded and the mean was calculated. The mean ENoG value, which indicates the degree of facial nerve degeneration, was $60.99 \pm 19.40 \%$.

Bithermal caloric testing was performed in dizzy patients using water at temperatures of $44^{\circ} \mathrm{C}$ and $30^{\circ} \mathrm{C}$ and canal paresis (CP) was calculated using Jonkee's formula [9]. CP exceeding $25 \%$ was considered to be abnormal.

MRI to rule out a central lesion was performed in all patients; 44 patients who visited the ER underwent diffusion MRI, and MRI of the internal auditory canal with and without contrast was assessed in the 54 patients who visited the outpatient clinic. The enhancement of the facial nerve was defined if each segment showed a hyperintense signal on contrast-enhanced T1-weighted MRI compared to the pre-contrast image.

For dizziness, spontaneous nystagmus (SN) and head-shaking nystagmus (HSN) were assessed in all dizzy patients using infrared goggles (SLMED, Seoul, Korea) [9].

\section{Treatment regimens}

All patients were treated with oral methylprednisolone (MPD) (48 mg/day for 4 days, followed by a tapering dose of $8 \mathrm{mg}$ every 2 days). In patients weighing less than $60 \mathrm{~kg}, 0.8$ $\mathrm{mg} / \mathrm{kg}$ of MPD was prescribed for 4 days first and then tapered over time. In addition to MPD, 3,000 mg/day of oral valacyclovir was prescribed for 7 days for combined steroid-antiviral treatment. In patients aged $<12$ years, $40 \mathrm{mg} / \mathrm{kg} /$ day of acyclovir was administered intravenously for 5 days.

\section{Evaluation of severity of facial paralysis}

The degree of facial palsy was assessed using HB scale at the initial visit, and at weeks 2, 6, and 10-16 after treatment. 
Final recovery was defined as HB I or II at week 10-16.

\section{Statistical analysis}

To test for normality, the Shapiro-Wilk test was performed. Two-tailed Fisher's exact test and/or the chi-square analysis was used for comparison of nominal variables. Student's t test and the Mann-Whitney U test were conducted to compare continuous variables between two groups. To identify the prognostic factors of the recovery of facial paralysis, backward conditional logistic regression analysis was performed with variables such as dizziness, diagnosis, hearing thresholds in the affected side, cVEMP asymmetry ratio, ENoG value, and the initial HB grade. All statistical analyses were performed using IBM SPSS Statistics for Macintosh, version 24.0 (IBM Corp., Armonk, NY, USA), and p values $<0.05$ were considered statistically significant.

\section{Results}

\section{General characteristics of dizziness in acute peripheral facial palsy}

Of the 98 patients, 15 (15.3\%) had accompanying dizziness. We reviewed the medical records of 6 patients in which the nature of dizziness was fully described both in the hospitalization period and the outpatient clinic. Among the 6 patients, $83.3 \%(n=5)$ had rotatory vertigo and only 1 patient had non-rotatory lightheadedness. With respect to duration of dizziness, $66.6 \%(n=4)$ complained of continuous dizziness but $33.3 \%(n=2)$ had intermittent dizziness. With respect to aggravating factors, $50 \%(n=3)$ had no specific aggravating factors, and 2 patients $(33.3 \%)$ reported that dizziness was exacerbated when they lay down on the bed and then arose. Change of head position was the aggravating factor in 1 patient. Dizziness was accompanied by nausea and vomiting in $83.3 \%(n=5)$, and 1 patient had lightheadedness alone. The dizziness in all patients disappeared with conservative treatment during the hospitalization period and did not require further treatment.

Age, sex, affected side, recurrence, diabetes, hypertension, and the initial severity of facial paralysis were not different according to presence of dizziness $(p>0.05)$ (Table 1). However, 11 of 15 patients with dizziness $(73.3 \%)$ had pain around the ear; this was more frequent compared with the 32 of 83 non-dizzy patients $(38.6 \%)(p=0.012)$. In addition, the mean hearing threshold of patients with dizziness $(27.53 \pm 23.93 \mathrm{~dB})$ was worse than that of those without dizziness $(16.99 \pm 17.66$ dB) $(p=0.048)$.

\section{Results of otoneurologic examination in APFP patients with dizziness}

On initial bedside examination of dizzy patients, SN and HSN was observed in $80 \%(n=12)$ and $66.7 \%(n=10)$, respectively. SN was observed in 4 of 6 in the BP group (66.7\%) and 8 of 9 in the RHS group (88.9\%). The character of SN was horizontal-torsional nystagmus beating to healthy side in most patients; 1 patient in each group had horizontal nystagmus beating to the lesion side. HSN was augmented in all patients who had SN. The mean CP value of all patients with dizziness was $27.56 \pm 22.65 \%$. With respect to CP higher than $25 \%$ (abnormal), 1 of 6 patients in the BP group and 8 of 9 patients in the RHS group had abnormal CP; this difference was significant ( $p=0.011$, two-tailed Fisher's exact test).

Table 1. Patient characteristics according to accompanying dizziness

\begin{tabular}{lccc}
\hline \multicolumn{1}{c}{ Variables } & With dizziness $(\mathrm{n}=15)$ & Without dizziness $(\mathrm{n}=83)$ & p value \\
\hline Age (years) & $47.20 \pm 13.13$ & $43.82 \pm 16.37$ & 0.451 \\
Male/female & $10 / 5$ & $43 / 40$ & 0.288 \\
Diabetes mellitus ( $\mathrm{n})$ & 2 & 3 & 0.115 \\
Hypertension (n) & 2 & 12 & 0.909 \\
Right/left & $10 / 5$ & $39 / 44$ & 0.161 \\
Recurrence $(\mathrm{n})$ & $15 / 0$ & $81 / 2$ & 1.000 \\
Pain around ear, $\mathrm{n}(\%)$ & $11(73.3)$ & $32(38.6)$ & $0.012^{*}$ \\
PTA on the affected side (dB) & $27.53 \pm 23.93$ & $16.99 \pm 17.66$ & $0.048^{*}$ \\
PTA on the nonaffected side (dB) & $13.67 \pm 10.72$ & $11.65 \pm 10.02$ & 0.479 \\
HB grade at initial visit & $3.07 \pm 0.80$ & $3.00 \pm 0.75$ & 0.067 \\
HB grade at 2 weeks & $3.00 \pm 1.20$ & $2.64 \pm 1.13$ & 0.358 \\
HB grade at 6 weeks & $2.38 \pm 0.92$ & $1.77 \pm 0.93$ & 0.604 \\
HB grade at 10-16 weeks & $1.33 \pm 0.72$ & $1.28 \pm 0.63$ & 0.056 \\
Electroneuronography (\%) & $60.07 \pm 19.25$ & 0.284 \\
cVEMP asymmetry (\%) & $66.91 \pm 22.03$ & $21.54 \pm 14.75$ & 0.723 \\
\hline
\end{tabular}

*p<0.05. PTA: pure-tone average, HB grade: House-Brackmann grade, cVEMP: cervical vestibular-evoked myogenic potential 
The mean cVEMP asymmetry ratio in patients with dizziness was $23.37 \pm 18.63 \%$, and that in non-dizzy patients was $21.54 \pm 14.75 \%$, which was non-significant $(p=0.723)$. The mean ENoG value in patients with dizziness was $66.75 \pm$ $20.88 \%$ and that in non-dizzy patients was $60.09 \pm 19.17 \%$. This difference was also non-significant $(p=0.292)$.

On MRI, facial nerve enhancement was most commonly seen in the geniculate ganglion $(n=50 ; 92.6 \%)$, followed by the tympanic segment $(n=48 ; 88.9 \%)$, labyrinthine segment $(\mathrm{n}=47 ; 87.0 \%)$, meatal segment $(\mathrm{n}=46 ; 85.2 \%)$, and mastoid segment $(n=45 ; 83.3 \%)$. There was no significant difference in the enhancement of each segment according to accompanying dizziness or diagnosis $(p>0.05)$.

\section{Difference between BP and RHS}

No difference was found in age, affected side, the degree of facial paralysis at 6 and 10-16 weeks, and ENoG value between BP and RHS patients ( $p>0.05)$. RHS patients complained of dizziness more frequently: 6 of $69(8.7 \%)$ had dizziness in the BP group, while 9 of 29 in the RHS group $(31.0 \%)$ had dizziness ( $p=0.005$, chi-square $=7.860)$.

Furthermore, patients with RHS had more frequent pain around the ear $(p<0.001)$, worse facial grade at initial visit ( $p=$ 0.048 ) and 2 weeks $(p=0.001)$, worse hearing thresholds ( $p=$ $0.006)$, and higher cVEMP asymmetry ratio $(p=0.018)$ (Table 2$)$.

\section{Prognosis}

Backward, conditional regression revealed that initial degree of facial paralysis was independently associated with fi- nal recovery (odds ratio $=0.225,95 \%$ confidence interval $=$ $0.057-0.894, p=0.034$ ). However, dizziness, as well as other variables, was not a significant prognostic factor.

\section{Discussion}

In this study, approximately $15 \%$ of patients with APFP had associated dizziness. Regarding the nature of the dizziness, continuous, horizontal-torsional SN without aggravating factors was most common. It was frequently accompanied by nausea and vomiting, and disappeared in all patients within a week.

$\mathrm{VN}$ is the second most common cause of dizziness following benign paroxysmal positional vertigo, and the latent reactivation of herpes simplex type 1 is presumed to be the main cause of VN. Additionally, microvascular ischemia in the labyrinth is also associated with VN [14]. Patients with VN have horizontal-torsional SN beating to the healthy side, an abnormal head impulse test, and ipsilateral caloric weakness. In addition, VN is characterized by the absence of auditory symptoms. Although some findings in VN are similar to dizziness in APFP, the major difference was that patients with APFP-associated dizziness usually have worse hearing thresholds and faster recovery within a week.

A previous study reported that SN was observed in $37.3 \%$ of patients with BP and in $62.2 \%$ of patients with RHS [15]. In that study, no BP patients had subjective dizziness. Direct comparison between our patients and their data was impossible because bedside examination was not performed in patients without dizziness in the present study.

Table 2. Comparison of variables according to disease entity

\begin{tabular}{|c|c|c|c|}
\hline Variables & Bell's palsy $(n=69)$ & Ramsay Hunt syndrome $(n=29)$ & $p$ value \\
\hline Age (years) & $44.28 \pm 16.60$ & $44.48 \pm 14.38$ & 0.953 \\
\hline Male/female & $35 / 34$ & $10 / 19$ & 0.141 \\
\hline Diabetes mellitus ( $n$ ) & 5 & 0 & 0.318 \\
\hline Hypertension (n) & 11 & 3 & 0.470 \\
\hline Dizziness (n) & 6 & 9 & $0.005^{*}$ \\
\hline Right/left & $39 / 30$ & $10 / 19$ & 0.076 \\
\hline Recurrence (n) & 2 & 0 & 1.000 \\
\hline Pain around ear, n (\%) & $18(26.1)$ & $25(86.2)$ & $<0.001^{*}$ \\
\hline PTA on the affected side $(\mathrm{dB})$ & $15.19 \pm 17.45$ & $26.66 \pm 20.37$ & $0.006^{*}$ \\
\hline PTA on the nonaffected side $(\mathrm{dB})$ & $11.75 \pm 10.79$ & $12.45 \pm 8.43$ & 0.757 \\
\hline $\mathrm{HB}$ grade at initial visit & $2.91 \pm 0.74$ & $3.24 \pm 0.74$ & $0.048^{*}$ \\
\hline HB grade at 2 weeks & $2.45 \pm 1.04$ & $3.67 \pm 0.99$ & $0.001^{*}$ \\
\hline HB grade at 6 weeks & $1.76 \pm 0.95$ & $2.30 \pm 0.82$ & 0.102 \\
\hline HB grade at $10-16$ weeks & $1.25 \pm 0.60$ & $1.38 \pm 0.73$ & 0.352 \\
\hline Electroneuronography (\%) & $59.92 \pm 19.13$ & $63.42 \pm 20.20$ & 0.452 \\
\hline Caloric weakness (\%) & $23.67 \pm 25.73$ & $29.33 \pm 10.77$ & 0.562 \\
\hline cVEMP asymmetry (\%) & $19.67 \pm 13.67$ & $31.17 \pm 19.00$ & $0.018^{*}$ \\
\hline
\end{tabular}


According to the literature review, controversy exists as to whether dizziness is one of the prognostic factors in APFP. Our data showed that dizziness was not associated with final recovery. Consistent with our findings, a previous study demonstrated that there was no relationship between the degree of recovery and vestibular function test findings [15]. In a study of 345 patients with APFP, the Yanagihara score and the change of that score at 1 month, and ENoG values were significant prognostic factors [16]. However, dizziness was not. A study of 101 patients with RHS revealed that cochleovestibular symptoms were not related to prognosis and the degree of facial impairment was the most important factor [17].

In contrast, a large sample study of 1521 patients with BP reported that the presence of vertigo was related to incomplete recovery of HB II-V; thus, dizziness seemed to be an unfavorable prognostic factor [18]. A study of 81 patients with RHS reported that dizziness may be associated with more severe facial nerve degeneration because worse ENoG values were observed in dizzy patients [19]. However, we did not find any difference in ENoG values between patients with dizziness and those without dizziness.

Considering dizzy patients with APFP tended to have worse hearing thresholds irrespective of BP or RHS, these symptoms might be caused by cranial nerve (CN) VIII involvement, not just subjective symptoms. For RHS, hypotheses for explaining CN VIII involvement are as follows: 1) viral transmission through the perineural tissue in the internal auditory canal; 2) entrance of VZV into the labyrinth via the dehiscent facial nerve canal; or 3) reactivation of the VZV in the geniculate, spiral, and/or vestibular ganglion [20, 21]. However, the mechanism for BP remains as yet unclear. We suggest that zoster sine herpete misdiagnosed as BP might play a role but additional virologic evaluation is needed to test our hypothesis.

A recent study showed that intratympanic steroid injection leads to faster recovery in BP, although there was no significant difference in final recovery [22]. In fact, this is a widely used simple, noninvasive technique for treatment of various inner ear diseases such as sudden hearing loss, dizziness, and acute tinnitus. Thus, it might also be helpful for patients with APFP and CN VIII involvement.

On the other hand, abnormal cVEMP response was reported to be associated with poor prognosis [23]. Abnormal cVEMP response reflects the activity of the saccule and the inferior vestibular nerve (IVN). Thus, abnormal cVEMP response in APFP may imply severe involvement of both CN VII and VIII including the IVN, which is less often affected than the superior vestibular nerve (SVN) [23]. Recently, the same group also reported the feasibility of ocular VEMP (oVEMP) response in BP, which evaluates the function of the utricle and SVN. The latency and the amplitude of oVEMP were different according to whether complete recovery was achieved or not [24].

In contrast, we found that there was no difference in cVEMP asymmetry ratio according to presence of dizziness in the present study. Based on our finding, the question is raised about the clinical significance of the cVEMP response in APFP. Consistent with our finding, a previous study also reported that the cVEMP asymmetry ratio was not different between patients with BP and normal controls [25].

Next, we found that pain around the ear was more frequently present in patients with dizziness irrespective of diagnosis. Peitersen reported that postauricular pain occurred in $52 \%$ of patients with BP and the recovery to normal function was different according to the presence of this pain [26]. The authors' previous study demonstrated that pain around the ear that persisted more than 3 months from onset was associated with incomplete recovery, although initial pain did not affect the final recovery [8].

With these findings in mind, the mechanism of dizziness in APFP may be associated with the transient involvement of the vestibulocochlear nerve because APFP patients with dizziness tended to have concurrent worse hearing thresholds and pain around the ear. However, transient involvement of the eighth $\mathrm{CN}$ appears to be weakly correlated with the extent of facial nerve damage. RHS caused by a virulent VZV infection seems to have a greater chance of accompanying audio-vestibular symptoms than BP. But it is also possible that VZV may sometimes lead to BP without vesicular eruption and/or other unidentified etiologies. For example, vascular compromise might affect the vestibulocochlear nerve as well as the facial nerve, causing dizziness in these BP patients too.

In summary, dizziness itself is not a "strong" prognostic factor for recovery in APFP. In addition, APFP-related pain around the ear or hearing loss may be partly associated with prognosis but the impact seems to be weak. Audio-vestibular function testing, MRI, and electrophysiologic tests such as ENoG and cVEMP may be helpful to evaluate the degree of $\mathrm{CN}$ involvement. However, it is clear that the initial severity of facial nerve paralysis is the more important factor affecting the final prognosis, and was superior to other factors.

The present study has some limitations. In addition to the retrospective study design, the number of patients with APFPassociated dizziness was merely 15 . This could be overcome by a future prospective, multi-center study. Second, we only assessed SN and HSN during the conventional bedside examination of patients with dizziness. The head impulse test, which is one of the more common vestibular tests that would enable us to confirm the unilateral vestibular deafferentation, was ex- 
cluded due to the incomplete medical records. Third, we performed ENoG only one time. The ENoG value is one of the prognostic factors for recovery in BP as described earlier, and it may be changed depending on the progression of the disease and the timing of the evaluation [12]. However, our insurance does not cover multiple tests, so we were only able to obtain one ENoG value. This might produce misleading results of the ENoG for predicting final recovery.

In conclusion, patients with APFP may have dizziness in the early stage. However, in most cases, the dizziness is transient and seems to be unrelated to the final prognosis.

\section{Acknowledgments}

This research was supported by EMBRI Grants 2017-EMBRIDJ0002 for H.Y.L. from the Eulji University and by the Basic Science Research Program through the National Research Foundation of Korea (NRF), funded by the Ministry of Education (NRF-2017 R1C1B5017839) for H.Y.L.

\section{Conflicts of interest}

The authors have no financial conflicts of interest.

\section{REFERENCES}

1) Quant EC, Jeste SS, Muni RH, Cape AV, Bhussar MK, Peleg AY. The benefits of steroids versus steroids plus antivirals for treatment of Bell's palsy: a meta-analysis. BMJ [serial online] 2009 Sep 7 [cited 2017 December 15]; 339:b3354. Available from: https://doi.org/10.1136/ bmj.b3354.

2) Henkel K, Lange P, Eiffert H, Nau R, Spreer A. Infections in the differential diagnosis of Bell's palsy: a plea for performing CSF analysis. Infection 2017;45:147-55.

3) Murakami S, Mizobuchi M, Nakashiro Y, Doi T, Hato N, Yanagihara N. Bell palsy and herpes simplex virus: identification of viral DNA in endoneurial fluid and muscle. Ann Intern Med 1996;124(1 Pt 1):27-30.

4) Lee HY, Kim MG, Park DC, Park MS, Byun JY, Yeo SG. Zoster sine herpete causing facial palsy. Am J Otolaryngol 2012;33:565-71.

5) Baugh RF, Basura GJ, Ishii LE, Schwartz SR, Drumheller CM, Burkholder R, et al. Clinical practice guideline: Bell's Palsy executive summary. Otolaryngol Head Neck Surg 2013;149:656-63.

6) Lee HY, Byun JY, Park MS, Yeo SG. Steroid-antiviral treatment improves the recovery rate in patients with severe Bell's palsy. Am J Med 2013;126:336-41.

7) Monsanto RD, Bittencourt AG, Bobato Neto NJ, Beilke SC, Lorenzetti FT, Salomone R. Treatment and prognosis of facial palsy on ramsay hunt syndrome: results based on a review of the literature. Int Arch Otorhinolaryngol 2016;20:394-400.

8) Lee HY, Ryu EW, Park SW, Kim SJ, Yeo SG, Park MS. Analysis of associated symptoms of Bell's palsy. Korean J OtorhinolaryngolHead Neck Surg 2011;54:683-7.
9) Kim CH, Jeong KH, Ahn SH, Shin DH, Kim YW, Shin JE. Vibrationand hyperventilation-induced nystagmus in patients with Ramsay Hunt syndrome with vertigo. Otolaryngol Head Neck Surg 2015;152: 912-8.

10) Chung MS, Lee JH, Kim DY, Lim YM, Ahn JH, Sung YS, et al. The clinical significance of findings obtained on 3D-FLAIR MR imaging in patients with Ramsay-Hunt syndrome. Laryngoscope 2015;125: 950-5.

11) Martin-Sanz E, Rueda A, Esteban-Sanchez J, Yanes J, Rey-Martinez J, Sanz-Fernandez R. Vestibular restoration and adaptation in vestibular neuritis and ramsay hunt syndrome with vertigo. Otol Neurotol 2017;38:e203-8.

12) Kim CH, Choi JW, Han KJ, Lee YS, Shin JE. Direction-fixed and direction-changing positional nystagmus in Ramsay Hunt syndrome. Otol Neurotol 2018;39:e209-13.

13) Kim JC, Cha WW, Chang DS, Lee HY. The effect of intravenous dexamethasone on the nausea accompanying vestibular neuritis: a preliminary study. Clin Ther 2015;37:2536-42.

14) Jeong SH, Kim HJ, Kim JS. Vestibular neuritis. Semin Neurol 2013; 33:185-94.

15) Watanabe T, Suzuki M. Equilibrium test findings in patients with Bell's palsy. Auris Nasus Larynx 2006;33:143-7.

16) Morishima N, Yagi R, Shimizu K, Ota S. Prognostic factors of synkinesis after Bell's palsy and Ramsay Hunt syndrome. Auris Nasus Larynx 2013;40:431-4.

17) Coulson S, Croxson GR, Adams R, Oey V. Prognostic factors in herpes zoster oticus (Ramsay Hunt syndrome). Otol Neurotol 2011;32: 1025-30.

18) Kasse CA, Cruz OL, Leonhardt FD, Testa JR, Ferri RG, Viertler EY. The value of prognostic clinical data in Bell's palsy. Braz J Otorhinolaryngol 2005;71:454-8.

19) Shin DH, Kim BR, Shin JE, Kim CH. Clinical manifestations in patients with herpes zoster oticus. Eur Arch Otorhinolaryngol 2016; 273:1739-43.

20) Kuhweide R, Van de Steene V, Vlaminck S, Casselman JW. Ramsay Hunt syndrome: pathophysiology of cochleovestibular symptoms. J Laryngol Otol 2002;116:844-8.

21) Murakami S, Nakashiro Y, Mizobuchi M, Hato N, Honda N, Gyo K. Varicella-zoster virus distribution in Ramsay Hunt syndrome revealed by polymerase chain reaction. Acta Otolaryngol 1998;118:145-9.

22) Chung JH, Park CW, Lee SH, Kim BS, Cho SH, Kim HY, et al. Intratympanic steroid injection for Bell's palsy: preliminary randomized controlled study. Otol Neurotol 2014;35:1673-8.

23) Chung JH, Lee SK, Kim SH, Yeo SG, Park MS, Byun JY. Neurotological parameters and prognosis of Bell's palsy patients. Audiol Neurootol 2015;20:117-21.

24) Yang CW, Kim SH, Yeo SG, Park MS, Byun JY. Ocular vestibular evoked myogenic potential testing for the prognosis of Bell's palsy. Acta Otolaryngol 2017;137:221-4.

25) Krbot Skoric M, Adamec I, Habek M. Vestibular evoked myogenic potentials in Bell's palsy. Neurol Sci 2014;35:1599-602.

26) Peitersen E. Bell's palsy: the spontaneous course of 2,500 peripheral facial nerve palsies of different etiologies. Acta Otolaryngol Suppl 2002;549:4-30. 